\title{
FIRST OBSERVATIONS WITH THE BONN WIDE-FIELD PHOTOMETER AND POLARIMETER (WWFPP): SURFACE POLARIMETRY OF THE CRAB NEBULA
}

\author{
TH. LEIBER ${ }^{1}$ and K. REIF ${ }^{2}$ \\ ${ }^{1}$ Sternwarte Universität Bonn, Auf dem Hügel 71, 53121 Bonn, Germany \\ ${ }^{2}$ Radioastronomisches Institut Universität Bonn, Germany
}

\begin{abstract}
To test the performance of the WWFPP in polarimetric mode, we performed surface polarimetry of the Crab nebula in February 1993 using the Asiago $1.82 \mathrm{~m}$ telescope of the 'Osservatorio Astronomico di Padova'. The polarimeter is a new design and enables us to do simultaneous wide-field polarimetry (15 x 15 arcmin field with 0.43 arcsec/pixel) of four polarization angles using a twin Wollaston prism or simultaneous polarimetry of two angles using a double wedge plate with orthogonal sheet polarizers in combination with an achromatic half wave plate. For the observations described here we used the twin Wollaston. The advantages of this design are independency of fluctuations of atmospheric transmission and identical seeing for one set of Stokes parameters. The results of the observations are in agreement with results of other investigators who performed surface polarimetry of the Crab Nebula.
\end{abstract}

\section{Introduction}

The accuracy of surface photometry of extended objects (e.g. of the larger galaxies of the local group) always suffer from problems in estimation of the background because of the small field of the usual CCD cameras. With surface polarimetry the problems are even more serious. Careful removal of the sky background and foreground polarization are necessary to obtain meaningful results and require a large field of view combined with sufficient sampling. Moreover to obtain the linear polarization it is required to observe the object with at least three different polarization angles. Most imaging polarimeters use a polarizing beam splitter or sheet polarizers so that multiple exposures are necessary to obtain the linear polarization. Because of the usually low degree of polarization, long exposure times are required so that atmospheric transparency and the seeing can change during the observations which renders the data reduction. To overcome these problems we built a focal-reducing camera designed for $\mathrm{f} / 8 \mathrm{RC}$-telescopes. The system consists of a focal reducer (reduction 0.45 ) and a CCD detector with an $2048^{2} 15 \mu$ pixel LORAL FA2048 CCD. The instrument is described in more detail in Reif et al. (1993). For example at the $1.23 \mathrm{~m}$ telescope at Calar Alto the field of view is $24 \times 24 \mathrm{arcmin}$ with $0.7 \mathrm{arcsec} / \mathrm{pixel}$. 


\section{The Polarimeter}

The polarimeter enables us to choose between two observing modes, the twin Wollaston or a double wedge plate with two orthogonal sheet polarizers in combination with a remote controlled rotatable achromatic halfwave plate. These components are located in the collimated beam between collimator and lens. The twin Wollaston has a beam separation of one degree and forms a fourfold image. The four images have $0,45,90$ and 135 degrees of polarization direction. The double wedge (beam separation two degrees) forms a double image having polarization directions of 0 and 90 degrees. The halfwave plate is then used to obtain the $\mathbf{4 5}$ and $\mathbf{1 3 5}$ degree angles with another exposure. Furthermore the halfwave plate can be used to get an unpolarized flat field by adding images obtained at positions of 22.5 degree steps of the halfwave plate. To avoid confusion we use a remote controlled mask in the focal plane of the telescope. For the twin Wollaston we use a mask with square holes (1.7 $\times 1.7$ arcmin on the sky) and for the double wedge we use a mask with stripes ( $4.9 \times 24$ arcmin on the sky).

\section{Observation}

To test the performance of the twin Wollaston polarimeter we observed the Crab Nebula during February 1993 at the $1.83 \mathrm{~m}$ telescope of the Osservatorio Astronomico di Padova. At this $\mathrm{f} / 9$ telescope we had a $15 \times 15$ arcmin field of view with 0.43 arcsec/pixel. During the observations the weather was nonphotometric with seeing of 4 arcsec. Since the twin Wollaston produces a fourfold image it is necessary to use four different mask positions to cover the whole field. For each mask position we took two $900 \mathrm{sec}$ exposures using a V filter.

For the image processing we use IRAF, the Image Reduction and Analysis Facility of the NOAO. All tasks for image aligning and combining are contained within this package. Every fourfold image is split into four separate images by multiplying it with a bimodal image of the mask obtained without the polarizer. It is created by filling the exposed sections with $1 \mathrm{~s}$ and the unexposed sections with 0 s. Subsequently these images are aligned to a common grid using a spline interpolation. The differences of the 0 and 90 degree respectively 45 and 135 degree images give the Stokes parameters $Q$ and $U$. The sum of these images gives the total intensity. Subtraction of the background sky is easy because of the large field-of-view.

The resulting images of total polarization intensity and degree of polarization agree very well with the results of other investigators (Hickson \& van den Bergh 1990). We find a maximum polarization of $50 \%$ that is lower than in Hickson \& van den Bergh (1990). This is maybe due to 4 arcsec seeing and additional line emission in the broad $V$ filter. Furthermore our $1800 \mathrm{sec}$ exposures show additional polarized intensity at the western bay and a filament southward of the eastern bay. The resulting image looks rather symmetric although the western features are fainter than the eastern ones.

The observations at Asiago showed the capabilities of WWFPP. Using the twin Wollaston polarimeter it is possible to perform wide-field surface polarimetry even under moderate conditions. 


\section{Acknowledgements}

The instrument has been realized in part through grant 05 5BN414 of the Verbundforschung Astronomie/Astrophysik, grant Bo 779/6 of the Deutsche Forschungsgemeinschaft and grant MWF IVA5 of the Land Nordrhein-Westfalen. The control electronics for the filter wheels was designed and built at the MPIA, Heidelberg. We would like to thank the Osservatorio Astronomico di Padova for generous allocation of observing time at the $1.82 \mathrm{~m}$ Asiago telescope.

\section{References}

Hickson, P. and van den Bergh, S., 1990. Astrophys. J, 365, 224.

Reif, K., Bagschik, K., Gebler, K.-H., Herkendell, W., Kohley, R., Leiber, Th., Müller, Ph., Pohlmann, Th. and Poschmann, H., 1993. In IAU Symp. 161, 'Astronomy from Wide-Field Imaging'. These proceedings. 